\title{
PHOTOGRAMMETRY IN EXPERIMENTS FOR HYDROGEOLOGICAL RISK ASSESSMENT
}

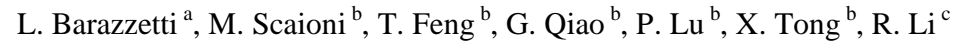 \\ ${ }^{a}$ Politecnico di Milano, Dept. of Architecture, Built Environment and Construction Engineering (ABC), \\ via M. d'Oggiono 18/a, 23900 Lecco, Italy - email: luigi.barazzetti@ polimi.it \\ ${ }^{\mathrm{b}}$ Tongji University, College of Surveying and Geo-Informatics \\ Center for Spatial Information Science and Sustainable Development Applications \\ 1239 Siping Road, 200092 Shanghai, P.R. China \\ email: [marco, fengtiantian, qiaogang, luping, xhtong]@tongji.edu.cn \\ ${ }^{\mathrm{c}}$ The Ohio State University, Mapping and GIS Lab \\ 470 Hitchcock Hall, 2070 Neil Avenue, Columbus, OH 43210-1275, The United States - email: li282@osu.edu
}

KEY WORDS: Hydrogeology, Image Metrology, Modeling, Photogrammetry, Risk Assessment, Simulations

\begin{abstract}
:
The construction of scaled-down simulation platforms is largely used to support investigations for the assessment of hydrological risk. Achieved outcomes can be integrated and assimilated to numerical analyses for the study of unstable slope collapse, debris transport, and hydrological modeling in general. During design of such simulation platforms, a relevant role has to be given to the spatial sensor network (SSN) to deploy, which is in charge of collecting geo-referenced, quantitative information during experiments. Photogrammetry (including 3D imaging sensors) can play an important role in SSN owing to its capability of collecting 2D images and 3D point clouds data covering wide surfaces without any contact. Different kinds of metric measurements can be then extracted from datasets. The aim of this paper is to give an overview and some examples on the potential of photogrammetry in hydrogeological experiments. After a general introduction on a few preliminary issues (sensors, calibration, ground reference, usage of imaging or ranging sensors), potential applications are classified into 2D and 3D categories. Examples are focused on a scaled-down landslide simulation platform developed at Tongji University (Shanghai, P.R. China).
\end{abstract}

\section{INTRODUCTION}

\subsection{Measurements in hydrogeological simulations}

The construction of scaled-down simulation models is largely used to support investigations for the assessment of hydrological risk. Achieved outcomes can be integrated and assimilated into numerical analyses for the study of unstable slope collapse ( $\mathrm{Lu}$ et al., 2012), debris transport process (Pozzoli, at al. 2004), shallow landslide triggering (Roncella et al., 2004), validation of hydrogeological numerical models, and the like.

The workflow in Figure 1 tries to resume the different levels of processes that can be observed during an experiment by using suitable sensors. The triggering factors are introduced to simulate natural or anthropogenic phenomena. For example, the load of a foundation over ground can be artificially reproduced by using a vertical loading machine. The progressive load can be measured during the experiment. In the case of rainfall induced landslides, water is distributed over a scaled-down model of a slope or inside the soil to simulate underground water circulation. In the case of loading, the triggering source is direct, while in the case of artificial rainfall is indirect. The triggering factors usually result in some final effects, e.g. the collapse of a slope or a specimen or the accumulation of debris and alluvium along the riverbed. Some quantities can be directly observed to be related to the final effects, like displacements or mass of accumulated material. In many cases, also some intermediate processes can be observed, establishing an indirect link between triggering factors and final effects. An example of such quantities is the soil pore water pressure that can be measured during a landslide simulation experiment.

In the setup of a simulation platform, a relevant role has to be given to the spatial sensor network (SSN), which is in charge of collecting geo-referenced, quantitative information during the experiments.

\begin{tabular}{|c|c|c|c|}
\hline PROCESS & \multicolumn{2}{|c|}{ OBSERVABLES } & SENSORS \\
\hline \multirow{2}{*}{ 息 } & \multicolumn{2}{|c|}{ DIRECT: loading } & $\begin{array}{l}\text { Direct reading from } \\
\text { actuators }\end{array}$ \\
\hline & \multicolumn{2}{|c|}{ INDIRECT: rainfall } & $\begin{array}{l}\text { Indirect readings } \\
\text { from sensors (e.g., } \\
\text { rainfall gauge) }\end{array}$ \\
\hline 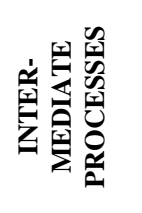 & None & $\begin{array}{l}\text { Pore water } \\
\text { pressure, } \\
\text { Osmotic } \\
\text { pressure, } \\
\text { Inner } \\
\text { stress,... }\end{array}$ & Direct reading \\
\hline \multirow{3}{*}{ 这空 } & \multirow{2}{*}{\multicolumn{2}{|c|}{ Displacements }} & $\begin{array}{l}\text { Pointwise meas. } \\
\text { (contact/remote) }\end{array}$ \\
\hline & & & $\begin{array}{l}\text { Area-based meas. } \\
\quad \text { (remote) }\end{array}$ \\
\hline & \multicolumn{2}{|c|}{ Debris accumulation } & $\begin{array}{c}\text { Collection of } \\
\text { accumulated } \\
\text { material }\end{array}$ \\
\hline
\end{tabular}

Figure 1. Workflow of possible processes and observables in experiments for hydrogeological simulations. 
The completeness of observations allows one to maximize the amount of information data achievable from a single or a set of tests. Results can be assimilated to numerical models to better estimate their parameters. A SSN also comprehends the ICT infrastructure as well as the devices for visualization, data storage and analysis.

Limiting here the discussion to the sensors, these can be classified into contact and remote categories. The first group collects all the ones that can record geometrical (spatial position, relative displacements, local deformations, rotations), geotechnical, hydraulic, and environmental parameters (e.g., weather, rainfall, etc.). The second group is based on noncontact sensors (or remote), whose aim is chiefly to give an overview on those processes occurring over a large surface of the experiment body, either from a qualitative (e.g., a video of the experiment run) and qualitative side. While contact sensors usually collect real-time information and can be used for forecasting purpose, remote sensors are chiefly adopted at the post-processing stage.

Photogrammetry (including also 3D scanning and 3D imaging sensors - see Luhmann et al., 2011; Remondino and Stoppa 2013) may play an important role in the 'remote sensor' subsection of the SSN. Indeed, it can provide information concerning $2 \mathrm{D}$ and $3 \mathrm{D}$ modifications of surfaces with highfrequency, accuracy and spatial resolution.

The paper would like to give an overview on the existing photogrammetric techniques that can be used in scaled-down simulation platforms. Moreover, some implementations carried out at Tongji University (Shanghai, P.R. China), also in cooperation with Politecnico di Milano (Italy) are presented and discussed in the following.

\subsection{Photogrammetry for laboratory experiments}

Photogrammetric techniques have been widely exploited for measurements in laboratory experiments on construction materials (see, e.g. Maas and Hampel, 2006; Barazzetti and Scaioni 2009; 2010; Roncella et al., 2012; Fedele et al., 2014). In particular, the diffusion of digital cameras and the increase of automation in modern digital photogrammetry fostered its application in this field with respect to the era of film cameras. In fact, the most important improvement in the digital era does not mainly consist in the better precision achievable (see Fraser, 1992, for example) but in the simplification and budgetary sustainability of its application. No special and expensive cameras are still needed, but a quite standard single lens reflex (SLR) digital camera can be used after calibration. Furthermore, all processing stages can be done using ordinary computers, without requiring optical-mechanics apparatus like in the past. Automation of both orientation and calibration stages has simplified and reduced the work the operator should do (Barazzetti et al., 2011). The stereoscopic plotting, that was the only way to derive 3D surfaces from analogue photos, has been replaced by automatic digital surface reconstruction, which works well in many applications.

On the other hand, the diffusion of digital techniques and the availability of low-cost photogrammetric software packages allowed everybody to accomplish photogrammetric projects where high precision is required, but the quality of results still depends on the experience and the personal background of the users.
Photogrammetry shows typical advantages of non-contact techniques, offering the chance to evaluate displacements or changes over larger areas rather than on single points like contact sensors do. Moreover, in the case high-dynamic phenomena should be investigated, like debris flow runouts, images can be acquired using high-speed video-cameras.

Modern photogrammetry does not only account for standard cameras, but might take advantage of the so called active $3 D$ imaging sensors. These are able to directly collect 3D data, unlike photogrammetry which needs to intersect rays from two known camera stations.

In experiments for testing hydrogeological models, usually a process is simulated at smaller scale than in reality. This can be a rainfall- or earthquake-induced landslide on a slope (see, e.g., Fukuzono, 1990), hydraulic model experiments (see Kabdasli et al., 2004), or triggering a shallow landslide on a flume (Roncella et al., 2004). Photogrammetry can be included in the SSN for the observation of the external surfaces of specimen, including (i) changes of the shape (deformations, loss and deposit of material), (ii) point or feature displacement tracking, and (iii) crack analysis (detection and measurement). All photogrammetric measurements are included in the 'Final Effects' phase shown in Figure 1. Various processes can be observed within a different approach if their domain can be approximately in $2 \mathrm{D}$ or $3 \mathrm{D}$ space. This option will involve a different geometric model and then a diverse geometry for image acquisition, as discussed in Section 3.

\subsection{The landslide simulation platform at Tongji University}

A scaled-down platform for the simulation of rainfall-induced landslides has been established in the campus of Tongji University, mainly for testing a SSN to be used in a real-scene environment (Qiao et al., 2013). The scaled-down model (see Fig. 2) has been designed to reproduce inclination, soil layers and composition of a ground slope in Taziping (Sichuan province, P.R. China). In this site, an active landslide was triggered after the 'Wenchuan earthquake' on May 12, 2008. To emulate the real slope, the model has been divided into three sectors with different inclinations $\left(5^{\circ}, 15^{\circ}\right.$ and $\left.30^{\circ}\right)$. Two soil layers have been laid down. The lower, with a depth of $50 \mathrm{~cm}$ is composed of a mixture of gravel, clay and sand in proportion 1:1:5. The upper one, with a depth of $30 \mathrm{~cm}$, is composed of clay and sand in proportion 1:3.

The sensors used in the simulation platform are representative of the more extensive equipment to be adopted for real landslides observation. They can be broadly classified into: (i) contact sensors, including geotechnical sensors, inclinometers and accelerometers; (ii) remote sensors consisting in cameras able to capture images at different speed and resolution (a pair of synchronized high-speed mono-chromatic cameras DALSA Falcon 4M60, a pair of low-speed SLR colour cameras Nikon D200 cameras, and one infrared video-camera for surveillance); and (iii) environmental sensors (weather station and a raingauge).

All sensors installed on the simulation platform are connected to a data acquisition unit, which synchronizes data gathering and deals with data broadcasting to a server station located in the university offices at about $800 \mathrm{~m}$ away. Here a database has been designed and implemented to store all recorded signals. 


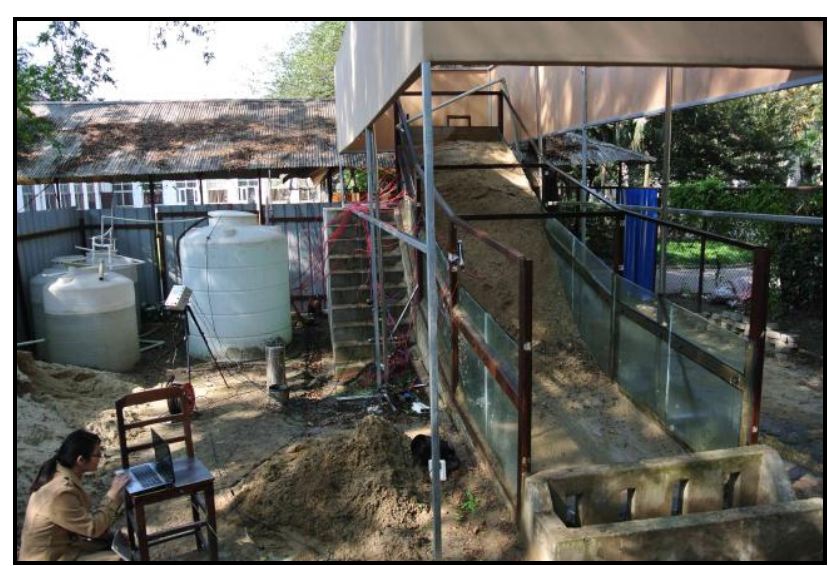

Figure 2. The landslide simulation platform established at Tongji University, Shanghai.

Data retrieving can be performed in real-time for visualization purpose. More details on different aspects of this project can be found in Feng et al. (2012) and Scaioni et al. (2012; 2013).

\section{REMOTE SENSORS}

\subsection{Digital cameras}

Digital cameras are the main sensors adopted in photogrammetry. While in the past only special metric or semimetric cameras were used, modern digital photogrammetry can be afforded with standard consumer cameras. Photogrammetry is based on measuring point coordinates on the images. This task requires establishing a reference system in the image that can be defined on the basis of the regular pixel grid. Consequently, other kinds of references used in old photogrammetric film cameras (like fiducial marks or 'réseaux') are not required any longer. A procedure called camera calibration allows the user to determine the basic parameters (the principal distance $c$ and the principal point coordinates $x_{0}$, $y_{0}$ ) that are required for modelling the inner formation process of an image (addressed to as inner orientation - IO). In addition, some additional parameters to compensate for lens distortion can be estimated. It is out of the scope of this paper to illustrate in depth he analytical models for camera calibration; the reader can refer to Luhmann et al. (2011) and Fraser (2013). We limit here to recall some remarks.

Calibration is strictly needed in 3D applications, because it directly affects the space intersection of rays from multiple camera stations. In 2D applications (Sect. 3) the need of calibration depends on the precision required in the project. Absolute errors in the images might reach some hundreds micrometres close to the sensor border, especially when a short focal length is used. The amount of this error has to be multiplied by the scale factor between image and real object to obtain the amount of error in the reconstruction. On the other hand, calibration can be avoided if relative displacements between two consecutive single images captured at different times are needed (Fedele et al., 2014). Exception is made if the very high precision must be reached, or with special algorithms like the one adopted in Section 3. As a rule of thumb, calibration is always suggested disregarding the specific application. Indeed, nowadays this task a very simple to accomplish, being available several low-cost (or also free) software packages allowing the rigorous photogrammetric calibration of any camera. The user has to capture with the same camera a set of images (\#8-15) from different positions, all depicting a set of coded targets (see Fig. 3). The resulting images are processed in fully automatic way to derive all calibration parameters to be used for the following photogrammetric application of the same camera. Focusing distance and focal length changes in zoom lenses affect calibration parameters. Consequently they must be kept at the same setup during both calibration and application stages. Two practical suggestions for a successful calibration (Remondino and Fraser, 2006) are: (i) to put targets on different planes and use convergent images; and (ii) not to use simplified calibration models, like the ones popular for many computer vision $(\mathrm{CV})$ applications which have not been thought for image metrology. Standard SLR cameras can acquire RGB (red-green-blue) images, while some industrial video-cameras for metrological applications can gather monochromatic intensity images only. Basically photogrammetry does not require colour images, except in the cases it is done for documentation or 3D visualization purpose (for example in Cultural Heritage). In the case subpixel precision is required, the user is suggested to work with the green channel only. Indeed, in CCD and CMOS sensors that are commonly adopted in RGB digital cameras, the elementary photodiodes do not capture all colours along with the same geometric scheme (called Bayer scheme). The colour which is captured in the more symmetric way is just green.

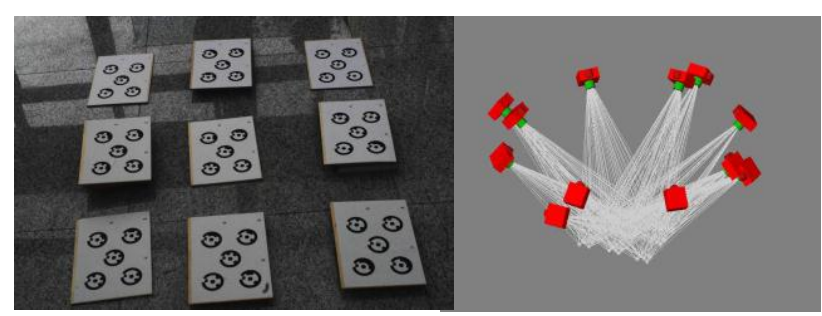

Figure 3. Examples of coded targets to be adopted for camera calibration and their spatial layout (left). Typical geometry of a block of convergent images used for camera calibration (right). The block should also include at least a couple of images taken with the camera rolled from landscape to portrait position.

\subsection{Active 3D imaging sensors}

An alternative approach to image-based photogrammetry relies on active $3 D$ imaging sensors. These can directly collect $3 \mathrm{D}$ surfaces, without needing any further processing stage, except that for registration of multiple 3D views and information extraction (Pirotti et al. 2013). Each technique may have its own calibration model and methodology.

Active 3D imaging sensors are ideal for reconstructing surfaces in the case the area is limited to few square metres and less. Their use is discouraged in favour of cameras when a high acquisition rate is needed, and when the aim is to extract features or to track points.

This category entails several different sensors featuring nonstandardized properties (like digital cameras basically do). Broadly speaking active 3D imaging sensors that can be used for close-range applications can be categorized into the following classes (see more details in Guidi et al., 2010): 
1. Triangulation scanners based on points, lines, patterns;

2. Time-of-Flight (ToF) range cameras; and

3. Phase-shift laser scanners.

Generally category (1) comprehends sensors that outperform the requirements of hydrogeological experiments. A point precision up to $0.1 \mathrm{~mm}$ and a point density of $1 \mathrm{~mm}$ grid can be reached. Sensors that go beyond these limits are available but their Fieldof-View (FoV) is usually limited to a few tens of square decimetres. In the case bigger static scenes should be reconstructed with higher precision and there is not any limitation on the surveying time, hand-held triangulation scanners can be used. However, usually they require putting some targets on the object surface.

If lower precision (at 1-2 cm level) and higher acquisition rate (more than $10 \mathrm{~Hz}$ ) are required, an interesting option is given by $T o F$ and triangulation cameras, including those in the gaming device category (e.g. the Microsoft Kinect ${ }^{\circledR}$ - see Chow et al., 2012). The advantage of using such device is their lowcost (about one hundred euros for gaming devices and up to one thousands for other systems), although they require the user to do sensor calibration on his own for performance improvement (Menna et al., 2011).

\subsection{Ground constraints}

In standard close-range photogrammetry some ground control points (GCP), whose coordinates have been measured independently (e.g. by using a theodolite), are usually placed on the surface of the object to reconstruct or close to it. The aim of GCPs is twofold: (1) to establish a global reference system (GRS) to compute point coordinates; and (2) to enforce the block geometry in the case many images are needed, or they spatial distribution is week; this is likely to happen if the block has an elongated shape, if the overlap between adjacent images is low, or if they feature large convergent angles (for more details see Barazzetti et al., 2011). Although problem (1) refers to both $2 \mathrm{D}$ and $3 \mathrm{D}$ cases, the second only concerns the $3 \mathrm{D}$ case; indeed, in $2 \mathrm{D}$ applications usually a single camera station is used.

Generally the use of GCPs makes photogrammetry more complicated, because it requires a further measurement instrument and the deployment of targets on the object. One of the advantages of modern digital photogrammetric techniques is that in many situations GCPs can be avoided. First of all, a redundant number of digital images can be collected to improve the computation of exterior orientation (EO). This consists in the position and attitude of each camera station with respect to a given GRS. Secondly, EO can be computed on the basis of tie points. These are corresponding points that are measured on more images by manual or automatic procedures (Barazzetti et al., 2011), but they do not direct measurement in the GRS. Thanks to a computational procedure called free-net bundle adjustment (Luhmann et al., 2011) all images can be oriented into an 'arbitrary' GRS, whose position with respect to the object is defined up to a 3D similarity transformation incorporating 3 shifts, 3 rotations in space, and one isotropic scale (7 parameters). In many applications, the reconstructed geometry of the object is needed only to compute the relative distances between points. Thanks to the knowledge of the real distance between two points, the global scale can be fixed. Moreover, the 'arbitrary' GRS can be also rotated by using a vertical element in the scene to define the $z$ axis, and a horizontal feature for the $x$ or $y$ axis.
In the case other $3 \mathrm{D}$ data like the ones gathered with an active 3D imaging instrument should be referred to the same GRS, some control points can be defined in the photogrammetric reconstruction for registration purpose.

In laboratory experiments very often only 2-3 fixed cameras are used for tracking point displacements and deformations over a small scene along time. The EO for such small configurations can be solved with easy without any GCP. In the case of multitemporal data acquisition, EO can be computed before the experiment, and then used for all epochs if the positions of all cameras are stable. In the case a bigger block is captured, for example for the reconstruction of the 3D surface of a hydraulic model of a river, the use of GCPs can be also avoided or at least reduced to a minimum if the following four rules are respected:

1. Redundant images;

2. Several tie points are measured on more than two images;

3. Large image overlaps between adjacent images; and

4. Small relative angles between adjacent images.

In any case, the acquisition scheme of large blocks should be assessed with major care. Numerical simulations of bundle adjustment can be used to ascertain the achievable theoretical accuracy during the design of the photogrammetric network.

\section{2D APPLICATIONS}

\subsection{Theoretical aspects}

When the surface to reconstruct is approximately flat, the transformation between image and object coordinates can be provided by a planar homography. This transformation is described by a $3 \times 3$ non-singular matrix $\mathbf{H}$ :

$$
\mathbf{x}=\left[\begin{array}{l}
x \\
y \\
1
\end{array}\right]=\left[\begin{array}{lll}
h_{1} & h_{2} & h_{3} \\
h_{4} & h_{5} & h_{6} \\
h_{7} & h_{8} & h_{9}
\end{array}\right]\left[\begin{array}{l}
X \\
Y \\
1
\end{array}\right]=\mathbf{H X},
$$

where vectors $\mathrm{x}=\left[\begin{array}{lll}x & y & 1\end{array}\right]^{\mathrm{T}}$ and $\mathrm{X}=\left[\begin{array}{lll}X & Y & 1\end{array}\right]^{\mathrm{T}}$ express image and object points in homogenous coordinates, respectively. In this case, the GRS has only two degree-of-freedom (DoF), being the object planar.

If the object is only approximately flat, the assumption of model (1) will lead to errors in the computed coordinate vector X. These errors can be evaluated with a simple formula that can be found in any handbook of photogrammetry.

Estimate of matrix $\mathbf{H}$ requires the determination of 8 parameters, because the scale of $\mathbf{H}$ can be arbitrarily set. A common method to do this is based on a set of at least four nonaligned GCPs whose coordinates are known in a 2D GRS and can be also measured in the image. Moreover, Eq. (1) shows that: (i) one image is enough for reconstructing a planar object; and (ii) the inverse transformation can be always worked out because $\mathbf{H}$ is non-singular. On the other hand, object coordinates $(\mathrm{X})$ are not always available. In this case, homography can be estimated as proposed in Barazzetti (2011) on the basis of the vanishing line of the plane (Hartley and Zissermann, 2006) coupled with constraints available from camera calibration. This information is sufficient to recover metric properties without acquiring metric data (e.g. known ratios of distances and angles, object points), except for an 
overall scale ambiguity that should be fixed by measuring a real distance.

\subsection{Example}

The image rectification method described in Barazzetti (2011) was applied to a set of images captured during a landslide simulation experiment ran in the platform described in subsection 1.3. Images were captured with a hand-held digital camera Nikon V1 (format $3872 \times 2592$ pixels, focal length 10 $\mathrm{mm}$, pixel size $3.4 \mu \mathrm{m}$ ) that was calibrated with a standard photogrammetric procedure. Image distortion was removed from all images that were resampled to obtain distortion-free images. As can be seen in the example reported in Figure 4, these images depict one of the lateral transparent windows of the simulation platform. Through this window the lateral ground profile during the landslide development can be recorded. Therefore images have to be registered in the same reference system for comparison. One image (the one in Fig. 4) has been rectified first, and then the resampled image used as reference (master) for the others. The procedure was based on the extraction of the parallel lines which form the frame around the window. These were then used to compute the vanishing line needed by the method. This also required the IO parameters derived from camera calibration to work out the initial homography $\mathbf{H}_{\mathbf{0}}$.

To automate the registration of the following images, all original images were matched with the SIFT operator (Lowe, 2004) in order to extract a dense set of tie points. SIFT operator is a feature detection algorithm which is invariant with respect to image shift, rotation, scale and brightness transformation. It also maintains a certain degree of stability under perspective and affine transformations. For this reason, it is usually adopted in photogrammetry and $\mathrm{CV}$ to find corresponding points between different images, to be exploited for the automatic orientation of 3D close-range blocks (see Barazzetti et al., 2010), but also for the registration of multi-temporal image sequences.

As the geometry of the block is quite poor (images have approximately the same perspective centres) a set of pixel points was extracted from the first rectified image and were used as GCPs for the rectification of the others. In this second case, these 'photogrammetric' GCPs allowed to compute the homography matrix $\mathbf{H}_{\mathbf{i}}$ of each $i$-th image of the sequence by using a standard point-based method. This provided the registration of the whole sequence made up of 20 images captured during an experiment, from which it was possible to draw the ground profile at different times.

\section{3D APPLICATIONS}

\subsection{Theoretical aspects}

If the object to survey cannot be approximated by a plane, the full perspective transformation from the object coordinates $\left(\mathrm{X}=\left[\begin{array}{lll}X & Y & Z\end{array}\right]^{\mathrm{T}}\right)$ of a point to its corresponding projection on the image $\left(\mathrm{x}=\mathrm{X}=\left[\begin{array}{lll}x & y & -c\end{array}\right]^{\mathrm{T}}\right)$ must be adopted. This is given by the collinearity equations:

$$
\left[\begin{array}{c}
x-x_{0}-\Delta x \\
y-y_{0}-\Delta y \\
-c
\end{array}\right]=\frac{1}{m} \mathbf{R}^{\mathrm{T}}\left(\mathrm{X}-\mathrm{X}_{0}\right)
$$

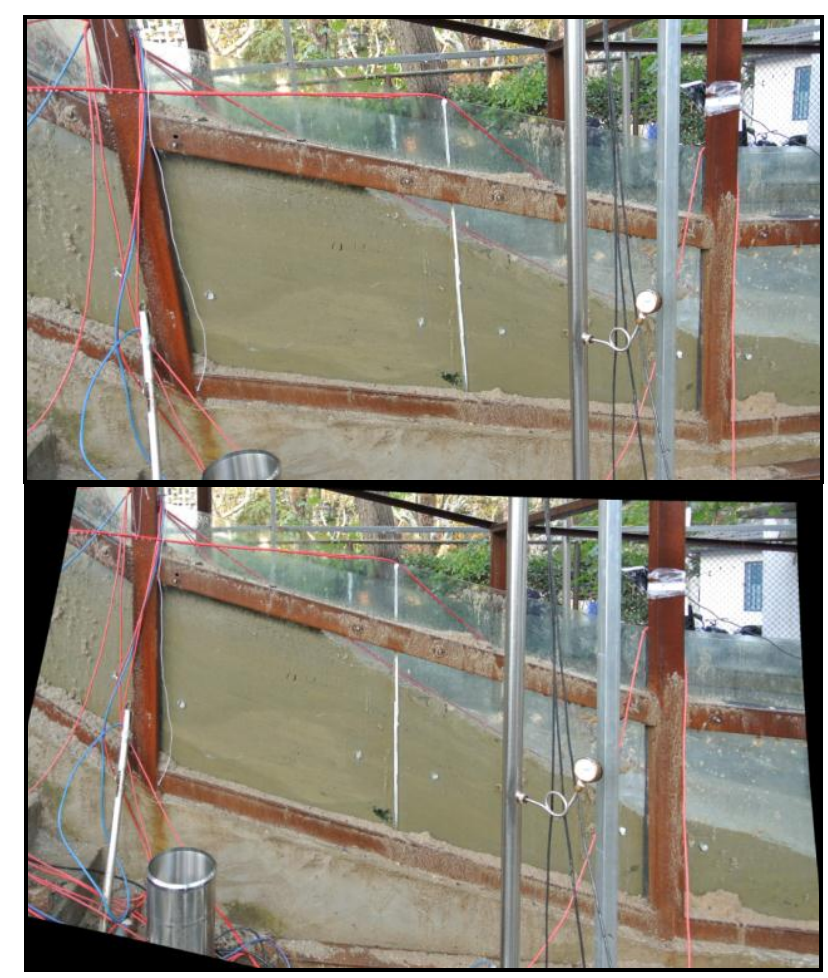

Figure 4. One of the images of the sequence (the one used as master) before (at top) and after rectification (at bottom).

where $\Delta x$ and $\Delta y$ express the corrections for image distortion to the image coordinates by means of the AP computed during camera calibration; the spatial rotation matrix $\mathbf{R}$ and the perspective centre vector $\mathrm{X}_{0}$ define the $\mathrm{EO}$ of the camera; the scale factor $m$ is cancelled out by dividing the first two lines of Eq. (2) by the third one. The collinearity model (2) can be used either for the computation of the unknown EO, given some GCP or using a free-net adjustment, and for the reconstruction of $3 \mathrm{D}$ coordinates of points, given the EO. Usually the IO parameters (including AP for lens distortion correction) are computed in a preliminary stage, but it is also possible to compute them along the reconstruction project (selfcalibration). The computation of EO is done through a Least Squares bundle adjustment, where tie point and GCP (if used) coordinates are included. Tie points can be manually measured by locating some natural features on the images, by using coded targets (like those used for camera calibration in Fig. 3), or by extracting key-points in automatic way with feature-based matching (FBM) algorithms (Barazzetti et al., 2010). The use of a rigorous Least Squares (LS) estimate in the bundle adjustment gives the opportunity to locate errors (if not too many) in image point coordinates and to estimate the theoretical accuracy of computed 3D points.

\subsection{Example}

The second technique implemented in the image-based monitoring system on the simulation platform relies on generation of a digital surface model (DSM) per each observation epoch, i.e. from each pair of stereo-images. A couple of SLR Nikon D200 cameras (format 2896x1944 pixels, focal length $35 \mathrm{~mm}$, pixel size $8.1 \mu \mathrm{m}$ ) were placed in front of the landslide scaled-down model in order to gather stereo- 
images during landslide experiments. Both cameras were kept in the same position during the whole experiment at a baseline of $1 \mathrm{~m}$. An acquisition rate of 2 frames/min was adopted. Before the experiment started, both cameras had been independently calibrated. After the experiment end, the first task was the computation of the EO of the stereo-camera system. This was accomplished by using some coded targets glued on the frame structure of the simulation platform, to be used as GCPs.

The photogrammetric procedure for DSM generation consists in the extraction of corresponding points from at least two images gathered at the same time. Thanks to the already known EO, the surface point extraction can be driven. The denser are the computed points the better will be the spatial resolution of the final DSM. For this reason, the extraction of surface points is done in automatic way by using image dense matching algorithms (Grün, 2012). In the latest years the number of available methods has grown exponentially. In metrological applications of close-range photogrammetry the so called areabased matching (ABM) techniques are usually adopted. If more than two cameras are available, a multi-photo approach (i.e., using more than two images) may increase the reliability of the extracted points as well as the chance to localize measurement errors. These methods may output a precise and very dense point cloud to describe the object's surface, but unfortunately are time consuming.

In this application a different approach has been followed, motivated by the need of computing volume changes but also to track point displacements on the slope surface. To this purpose, a set of key-points have been extracted by means of the SIFT algorithm (see Subsect. 3.2). As described in Feng et al. (2012), key-points have been matched along the sequence for reconstructing the point displacement field. Here the same set of key-points is matched across each synchronous image pair by using the procedure described in subsection 3.2, i.e., by exploiting the SIFT descriptors. 3D point coordinates can be computed thanks to forward intersection based on Eq. (2). A specific DSM can be reconstructed for each image pair. Thus, the changes of the slope surface during the experiment can be detected by comparing DSMs along time.

The comparison of two DSM deserves a comment. Unlike the point tracking which can be done with contact or topographic instruments or using an image-based method, a couple of DSMs cannot be compared in a point-wise manner. This is because the points used to generate a DSM at one epoch might be different from those used in the second. In addition, image matching can result in an irregular point cloud, having a different point density according to the local properties and texture of each portions of the object. To overcome these problems, every DSM is interpolated into regular a grid, whose coordinates are defined with respect to an assigned reference plane. The plane and the grid must be the same at both epochs. In this example, the average plane interpolating the reconstructed slope surface was selected as reference. A grid size of $1 \times 1 \mathrm{~cm}$ was adopted for interpolating points. The differences between the two surfaces were computed in correspondence of each grid node, yielding the displacement maps reported in Figure 5. The selection of the reference plane is really strategic, because only displacements along the normal direction to that can be evaluated. This involves that, in the case of more complex deformation processes, more reference planes should be established.

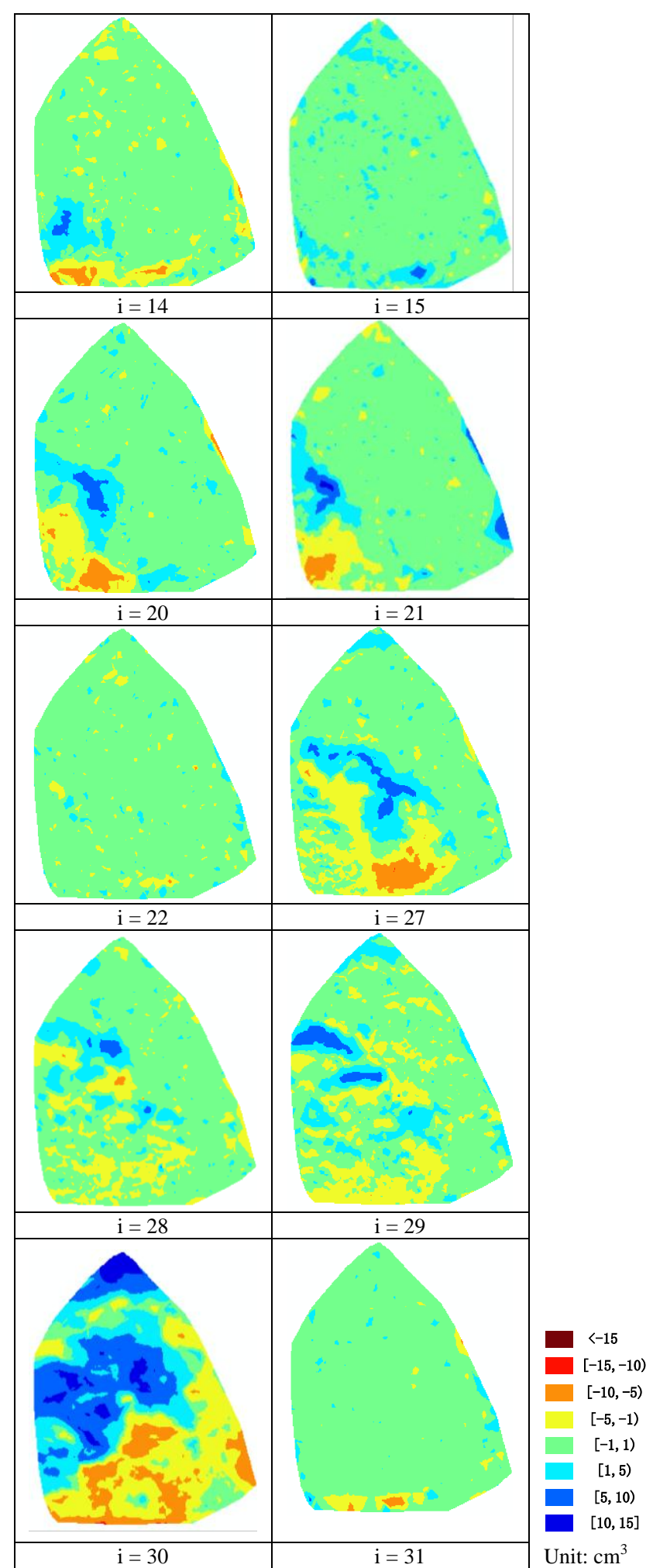

Figure 5. Landslide volumetric changes detected from the comparison of DSMs reconstructed at different epochs; here only the most representative epochs are illustrated. 


\section{CONCLUSIONS}

In this paper some applications of photogrammetry to gather data during a landslide simulation experiment have been reported. Such examples would demonstrate how this noncontact technique might offer different opportunities to retrieve both qualitative and quantitative information during a wide range of laboratory tests for hydrogeological risk assessment.

The application of photogrammetry is today simpler than in the past thanks to development of digital techniques and automation processes. The availability of 3D imaging sensors, also at low-cost, can be exploited to enrich and improve the information that can be obtained from purely image-based methods. For examples, in the case some features should be extracted or tracked along time, images are more convenient. In the case of surface reconstruction, the use of ranging devices might be simpler.

On the other hand, the impressive technological development does not replace the role of users, which must be aware of photogrammetric techniques and use them in a rigorous manner. This consideration also entails data interpretation. Photogrammetry provides mainly area-based information whose understanding might be different than that of traditional pointbased measurements. The cooperation between end-users and experts of photogrammetry has to be encouraged to improve the quality of results. Moreover, many potential applications cannot be afforded using off-the-shelf photogrammetric products, but they need tailoring existing algorithms or developing new research content.

\section{Acknowledgements}

This study is supported by the National High Technology Research and Development Program of China (No. 2012AA121302), the Basic National Research Program of China (No. 2013CB733204). This research partially benefited from the support of the FIRB 2010 National Research Program of the Italian Ministry of University and Research (MIUR).

\section{REFERENCES}

\section{References from Journals:}

Barazzetti, L., and M. Scaioni, 2009. Crack measurement: development, testing and applications of an automatic imagebased algorithm. ISPRS Journal of Photogrammetry and Remote Sensing, 64(3): 285-296.

Barazzetti, L., and M. Scaioni, 2010. Development and Implementation of Image-based Algorithms for Measurement of Deformations in Material Testing. Sensors, 10(8): 7469-7495.

Fedele, R., Scaioni, M., Barazzetti, L., Rosati, G., Biolzi, L., and P. Condoleo, 2014. Delamination tests on CFRP-reinforced masonry pillars: optical monitoring and mechanical modelling. Cement and Concrete Composites, Vol. 45, pp. 243-254, DOI:10.1016/j.cemconcomp.2013.10.006 [SCI].

Fraser, C.S., 1992. Photogrammetric measurement to one part in a million. Photogrammetric Engineering and Remote Sensing, 58(3): 305-309.

Fraser, C.S., 2013, Automatic camera calibration in Close Range Photogrammetry. Photogrammetric Engineering and Remote Sensing, 79:381-388.
Fukuzono, T., 1990. Recent Studies on Time Prediction of Slope Failure. Landslide News, 4: 9-12.

Grün, A., 2012. Development and Status of Image Matching in Photogrammetry. Photogrammetric Record, 27(137): 36-57.

Lowe, D.G., 2004. Distinctive Image Features from ScaleInvariant Keypoints. International Journal of Computer Vision, 60: $91-110$

Maas, H.G., and U. Hampel, 2006. Photogrammetric techniques in civil engineering material testing and structure monitoring. Photogrammetric Engineering and Remote Sensing, 72: 39-45.

Pirotti, F., Guarnieri, A., Vettore, A., 2013. State of the art of ground and aerial laser scanning technologies for highresolution topography of the earth surface. European Journal of Remote Sensing 46: 66-78.

Qiao, G., Lu, P., Scaioni, M., Xu, S., Tong, X., Feng, T., Wu, H., Chen, W., Tian, Y., Wang, W., and R. Li, 2013. Landslide Investigation with Remote Sensing and Sensor Network: From Susceptibility Mapping and Scaled-down Simulation towards in situ Sensor Network Design. Remote Sensing, 5(9): 4319-4346.

Scaioni, M., Lu, P., Feng, T., Chen, W., Wu, H., Tong, X., Wang, W., and R. Li, 2013. Analysis of spatial sensor network observations during landslide simulation experiments. European Journal of Environmental and Civil Engineering, 17(9): 802-825.

\section{References from Books:}

Guidi, G., Russo, M., and J.A. Beraldin, 2010. Acquisizione 3D e modellazione poligonale. McGraw-Hill, Milano, Italy, 466 pp. (in Italian).

Hartley, R., and A. Zisserman, 2006. Multiple View Geometry in Computer Vision. Cambridge University Press, UK.

Luhmann, T., Robson, S., Kyle, S., and I. Harley, 2011. Close Range Photogrammetry: Principles, Techniques and Applications. Whittles Publishing, Dunbeath, Caithness, Scotland (UK), 528 pp.

Pozzoli, A., Mussio, L., and M. Scaioni, 2004. A Project for the Survey of a Hydraulic Stream Confluence Model Based on a General Procedure for Three-Image Orientation. In: Commemorative Volume for the 60th birthday of Prof. Dr. Armin Grün, IGP ETH Zurich (Switzerland), pp. 201-208.

Remondino, F., and D. Stoppa, 2013. TOF Range-Imaging Cameras. Springer-Verlag, Germany, 240 pages.

\section{References from Other Literature:}

Barazzetti L., 2011. Planar metric rectification via parallelograms In: Proc. Int. Conf. 'Videometrics, Range Imaging, and Applications XI,' 23-26 May, Munich, Germany, Proc. of SPIE, Vol. 8085, No. 80850R, 12 pp. (e-doc).

Barazzetti L., Forlani G., Remondino, F., Roncella R., and M. Scaioni, 2011. Experiences and achievements in automated image sequence orientation for close-range photogrammetric projects. In: Proc. Int. Conf. 'Videometrics, Range Imaging, and Applications XI,' 23-26 May, Munich, Germany, Proc. of SPIE, Vol. 8085, No. 80850F, 13 pp. (e-doc).

Chow, J.C.K., Ang, K.D., Lichti, D.D., and W.F. Teskey, 2012. Performance analysis of a low-cost triangulation-based 3D camera: Microsoft Kinect system. In: The International Archives of the Photogrammetry, Remote Sensing and Spatial Information Sciences (IAPRS\&SIS), Vol. 39, Part B/5, pp. 175180.

Feng, T., Liu, X., Scaioni, M., Lin, X., and R. Li, 2012. Realtime Landslide Monitoring using Close-range Stereo Image 
Sequences Analysis. In: Proc. Int. Conf. ICSAI 2012, 19-21 May, Yantai, P.R. China, pp. 249-253.

Kabdasli, M.S., Celikoyan, T.M., Seker, D.Z., Aydingakko, A., 2004. Application of digital photogrammetry and image processing techniques for hydraulic model experiments. In: IAPRS\&SIS, Vol. 35, Part B/III, pp. 739-743.

Menna, F., Remondino, F., Battisti, R., and E. Nocerino, 2011. Geometric investigation of a gaming active device. In: Proc. Int. Conf. 'Videometrics, Range Imaging, and Applications XI,' 2326 May, Munich, Germany, Proc. of SPIE, Vol. 8085, No. 80850G, e-doc.

Remondino, F., and C.S. Fraser, 2006. Digital camera calibration methods: Considerations and comparisons. In: IAPRS\&SIS, Vol. 36, Part 5, pp. 266-272.

Roncella, R., Scaioni, M., and G. Forlani, 2004. Application of Digital Photogrammetry in Geotechnics. In: IAPRS\&SIS, Vol. 35, Part B/III, pp. 93-98.

Roncella, R., Romeo, E., Barazzetti, L., Gianinetto, M., and M. Scaioni, 2012. Comparative Analysis of Digital Image Correlation Techniques for In-plane Displacement Measurements. In: Proc. $5^{\text {th }}$ Int. Conf. 'Image and Signal Processing' (CISP 2012), 16-18 Oct., Chongqing, P.R. China, pp. 887-890.

Scaioni, M., Lu, P., Chen, W., Wu, H.B., Qiao, G., Feng, T., Wang, W., and R. Li, 2012. Wireless Sensor Network based Monitoring on a Landslide Simulation Platform. In: Proc. Int. Conf. WiCOM2012, 21-23 Sept, Shanghai, P.R. China, 4 pp. 